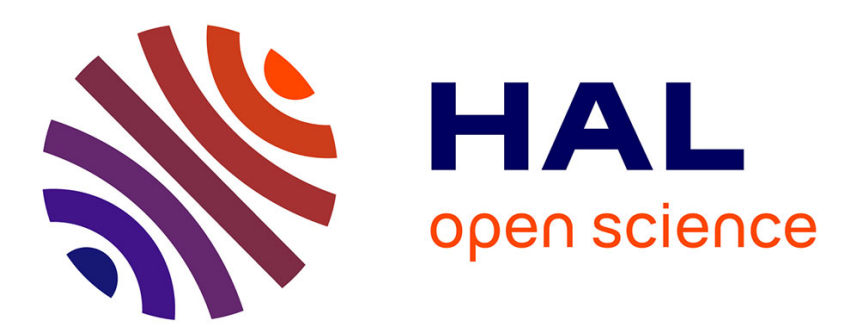

\title{
Numerical and experimental analyses of punch wear in the blanking of copper alloy thin sheet
}

Edouard Falconnet, Hamid Makich, Jérôme Chambert, Guy Monteil, Philippe Picart

\section{- To cite this version:}

Edouard Falconnet, Hamid Makich, Jérôme Chambert, Guy Monteil, Philippe Picart. Numerical and experimental analyses of punch wear in the blanking of copper alloy thin sheet. Wear, 2012, 296 (S1-2), pp.598 - 606. hal-02300235

\section{HAL Id: hal-02300235 \\ https://hal.science/hal-02300235}

Submitted on 29 Sep 2019

HAL is a multi-disciplinary open access archive for the deposit and dissemination of scientific research documents, whether they are published or not. The documents may come from teaching and research institutions in France or abroad, or from public or private research centers.
L'archive ouverte pluridisciplinaire HAL, est destinée au dépôt et à la diffusion de documents scientifiques de niveau recherche, publiés ou non, émanant des établissements d'enseignement et de recherche français ou étrangers, des laboratoires publics ou privés. 


\title{
Numerical and experimental analyses of punch wear in the blanking of copper alloy thin sheet
}

\author{
E. Falconnet ${ }^{\text {a }}$, H. Makich ${ }^{\text {a }}$, J. Chambert ${ }^{\text {b,* }}$, G. Monteil ${ }^{a}$, P. Picart ${ }^{\text {b }}$ \\ ${ }^{a}$ ENSMM, FEMTO-ST Institute, Department of Applied Mechanics, CNRS UMR 6174, 24 rue de l'Epitaphe, \\ 25000 Besançon, France \\ ${ }^{\mathrm{b}}$ University of Franche-Comté, FEMTO-ST Institute, Department of Applied Mechanics, CNRS UMR 6174, 24 \\ rue de l'Epitaphe, 25000 Besançon, France \\ * Corresponding author. Tel.: +33 3816660 25; fax: +33 381666700 . \\ E-mail address: jerome.chambert@univ-fcomte.fr (J. Chambert).
}

\begin{abstract}
This research on punch wear resulting from the blanking of copper alloy thin sheet has been conducted by means of experimental and numerical analyses. Firstly, the experimental method has consisted in measuring punch worn profiles from replicas, and secondly in obtaining the wear coefficient by using a specific tribometer. The numerical modelling of blanking process has been developed with the finite element method to compute the mechanical fields necessary to calculate wear. Thus, the Archard formulation for abrasive wear has been programmed to compute the wear depth and the resulting punch geometry. Finally the simulation results of wear prediction have been compared to experimental ones.
\end{abstract}

Keywords : Wear testing; Blanking; Copper alloy; Wear modelling; Finite element modelling 


\section{Introduction}

Among the shearing processes, blanking is one of the most frequently used due to its reliability and capability of mass production. The process is also very economical because it allows to easily obtain the appropriate size and shape of a large parts number. As in every production process, tools life time is of major concern for multiple reasons: the part quality mainly depends on tool wear, frequent tool changes slow down production time and increase maintenance costs. Therefore, predicting tool wear can be a key feature to improve the process efficiency.

For the last decades, wear in blanking has been the subject of several experimental researches, mainly to understand its impact on the product quality (burr height). Maeda et al. [1,2] have investigated blanking tool wear distribution and its influence on the process parameters (blanking force, energy, burr height). Hernandez et al. [3] have studied the effects of tool wear on the shearing mechanism and resulting form errors. Monteil et al. [4] have developed a direct method for measuring punch wear, based on selective activation technique, which allows to quantify in real time the influence of blanked material or lubrication. However, compared to other forming processes such as deep drawing [5,6] and hot forging [7], the combination of experimental and numerical methods to predict tools wear has been less investigated in blanking. Hambli [8] has developed a finite element (FE) model and has implemented an algorithm to predict punch wear during steel metal sheet blanking. The Archard formulation [9] for adhesive wear was considered to calculate wear depth on the punch, and a comparison with experimental results was performed. Hambli [10] has also proposed a theoretical approach to predict tool wear based on the evolution of the punch edge radius within Blanksoft, an industrial software dedicated to the optimization of sheet metal 
blanking processes. It is worth noting that the mentioned studies have been conducted with steel and stainless steel materials; less interest has been accorded to copper alloys. In the present paper, punch wear profile has been determined experimentally by a doublereplication method, and the wear coefficient of Archard formulation has been obtained by a specific tribometer. Then, FE simulation results have been used within a wear algorithm based on an Archard formulation applied to abrasive wear in order to predict the worn punch profile. The final section of this paper presents a comparison between experimental and numerical results of wear prediction.

\section{Experimental method}

The main objective of the experimental study conducted by Makich [11] has been to determine the influence of punch wear on the part quality during copper alloy thin-sheets blanking. A double-molding procedure has been developed to observe wear profiles, and a specific tribometer has been designed to determine the wear behaviour of several material couples.

\subsection{Blanking test description}

As shown in Fig. 1a, the blanking process consists in a metal sheet positioned over a die in which a punch passes through to plastically deform the metal until a crack propagates and leads to complete separation of the cut part. A sheet-holder is added to maintain the sheet during the travel of the punch to avoid elastic springback and movement of the sheet, thus increasing the quality of the cut edge profile. 

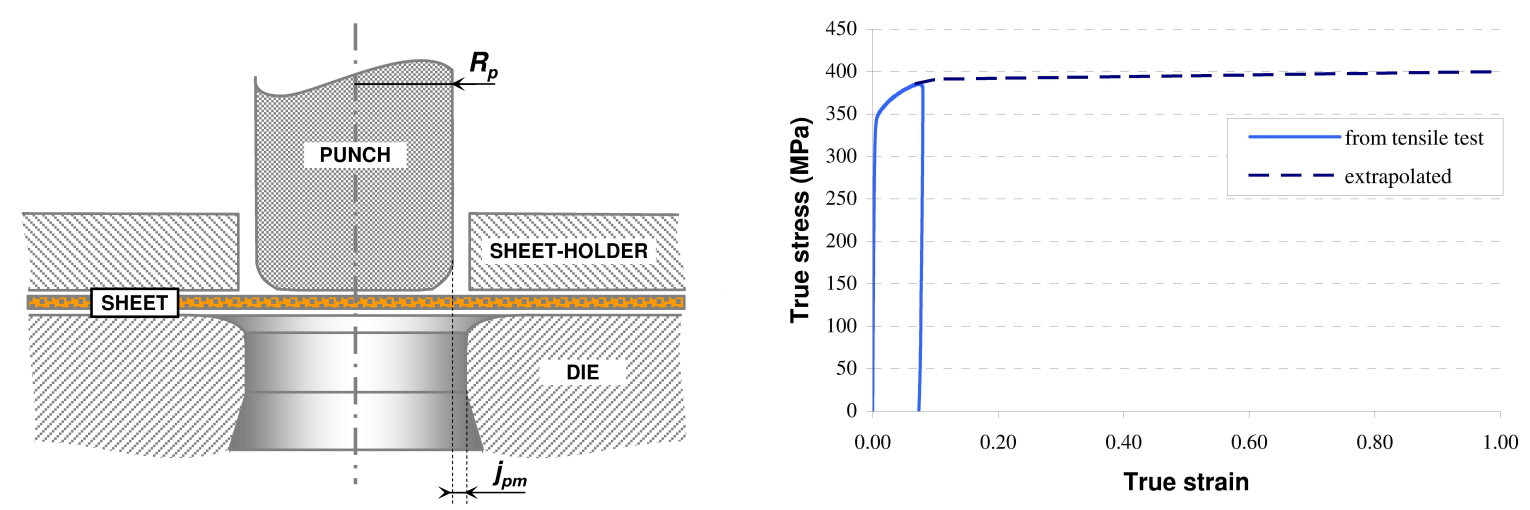

Fig 1: a) Configuration of the studied blanking process b) Tensile test curve of the blanked material

The blanking tests have been performed on a BRUDERER 50 tons press with a speed of 500 strokes/min. The principal geometrical data of the model is illustrated in Fig. 1a and listed in Table 1.

\begin{tabular}{llll}
\hline Parameter & Punch radius $R_{p}$ & Sheet thickness $e$ & Punch - die clearance $j_{p m}$ \\
Value & $1.85 \mathrm{~mm}$ & $254 \mu \mathrm{m}$ & $10 \mu \mathrm{m}$
\end{tabular}

Table 1. Main geometrical characteristics of the blanking process

In this study, the blanked material is a copper-iron alloy strip of $15 \mathrm{~mm}$ width and $0.254 \mathrm{~mm}$ nominal thickness. The mechanical properties of the blanked material have been obtained by a conventional tensile test. The true-stress - true-strain curve (Fig. 1b) has been approximated by the following equation:

$\sigma=\sigma_{0}+k \varepsilon^{n}$

The rational (true stress and strain) tensile test curve (Fig. 1b) has been linearly extrapolated to take account of large deformation plasticity within FE modelling. The chemical composition and mechanical properties are respectively provided in Table 2 and 3.

\begin{tabular}{lllll}
\hline Element & $\mathrm{Cu}$ & $\mathrm{Fe}$ & $\mathrm{P}$ & Others \\
wt.\% & $>99.61$ & $0.05-0.15$ & $0.025-0.04$ & $<0.2$
\end{tabular}

Table 2. Chemical composition of the blanked material 


\begin{tabular}{lll}
\hline Parameter & Symbol & Value \\
Young's modulus & $E$ & $121 \mathrm{GPa}$ \\
Poisson's ratio & $v$ & 0.34 \\
Yield strength & $\sigma_{0}$ & $332 \mathrm{MPa}$ \\
Material constant & $k$ & $221 \mathrm{MPa}$ \\
Strain hardening exponent & $n$ & 0.52 \\
\hline
\end{tabular}

Table 3. Mechanical properties of the blanked material

2.2 Determination of the punch wear profile

A double-print method has been developed in order to obtain the punch wear profile at different production stages without removing the punch from the mechanical press, and then not modifying the operating conditions. This in situ procedure of wear measurement by profilometry was divided into three major steps :

- Obtaining the imprint of the punch (without dismantling)

- Making a replica of the punch

- Measuring the edges of the replica by profilometry.

Observation of the punch by microscopy has revealed that the wear mechanism involved was purely abrasive.

\subsubsection{Imprint of the punch}

To carry out the imprint of the punch, a mold has been milled in a wedge block (Fig. 2a and 2b) to be filled with a volume of silicone resin (designated Coltène ${ }^{\circledR}$ President plus jet Light Body). The extremity of the punch has been immerged in this mold for a couple of minutes, according to the resin drying time. Impressions have been taken every 12,500 strokes of the punch. 

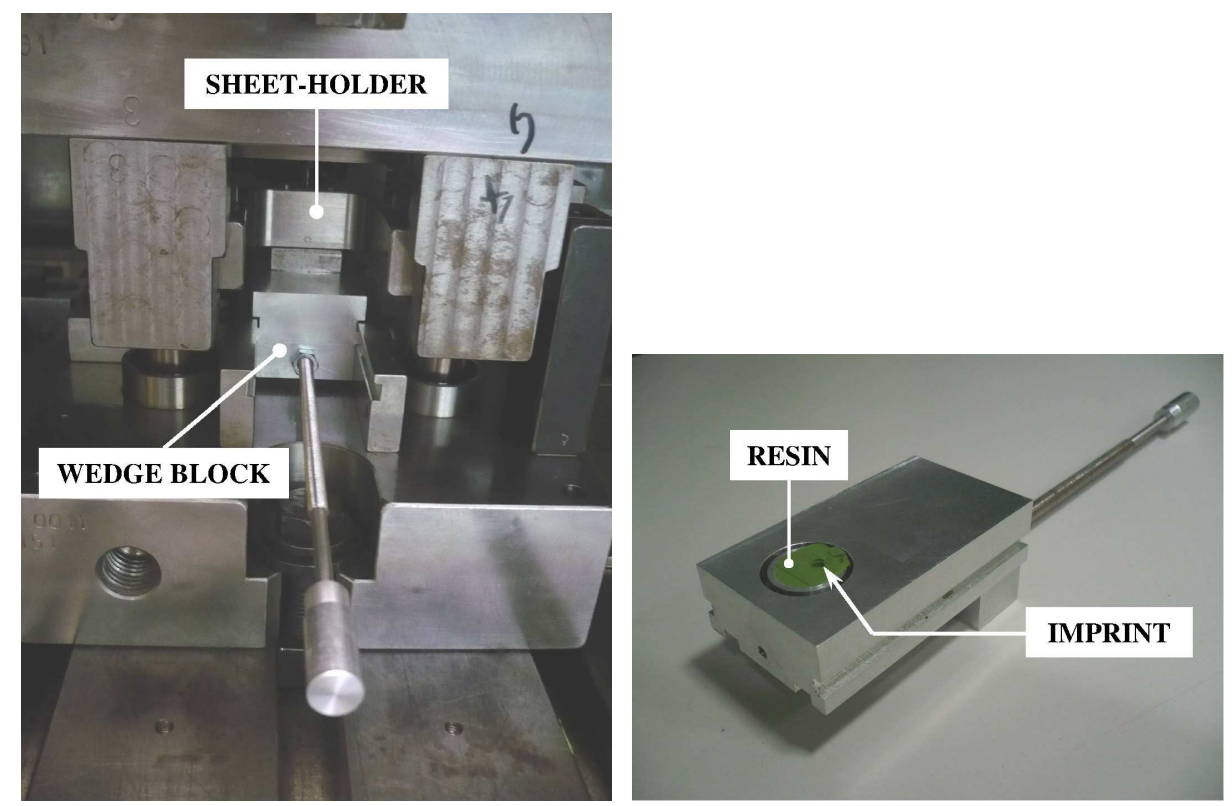

Fig. 2 : Experimental device used for producing an imprint of the punch : (a) global view on the mechanical press (b) detailed view of the wedge block

\subsubsection{Replica of the punch}

Once the impression of the punch has been performed, another silicon resin (called Rencast ${ }^{\circledR}$ FC 52 Isocyanate \& Polyol) compatible with the first one has been used to produce punch replica.

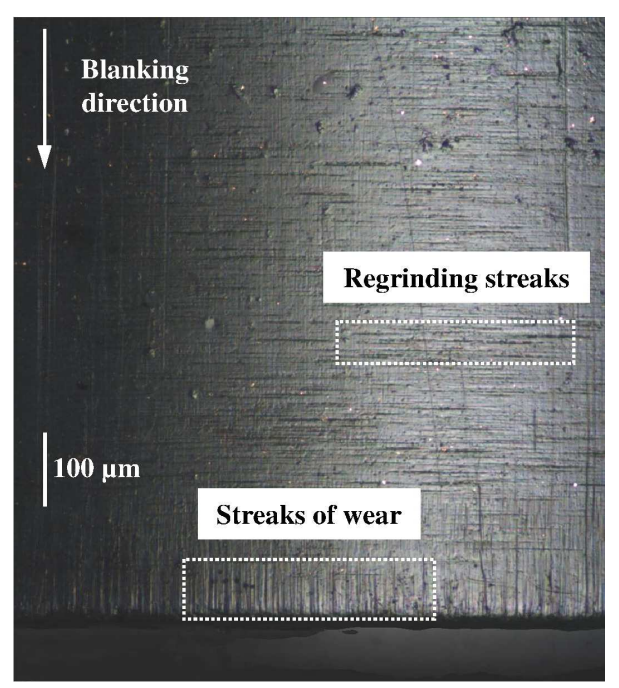

Fig. 3 : Replica of a punch after 787,500 strokes

Fig. 3 gives a detailed representation of a punch replica after 787,500 punch strokes. Besides the presence of abrasive wear streaks, the precision of this method allows to observe regrinding streaks. 


\subsubsection{Extraction of wear profiles}

When the replica of the punch has been carried out, a variable focus optical microscope (InfiniteFocus ${ }^{\circledR}$ ) has been used to extract 3D surfaces of the punch at four diametrically opposed locations. For each surface, an average profile has been determined and a representative state of wear for a given punch strokes number has been obtained by calculating an average profile from the four previous ones. Finally, a mathematical equation has been employed to fit this profile (Fig. 4), thus allowing to integrate the punch geometry in a numerical simulation. As already mentioned by Hernandez et al. [3], the worn punch profile may be decomposed in three zones: face wear, cutting edge wear and flank wear (Fig. 4).

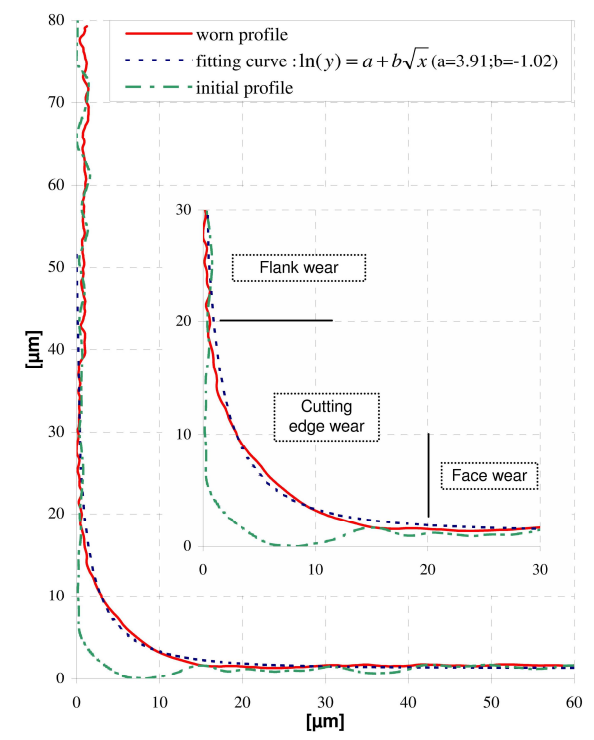

Fig. 4 : Initial profile, worn profile and corresponding fitting curve (766,500 punch strokes) from a punch replica with zoom on the cutting edge

In addition, the initial profile obtained after regrinding is shown on Fig. 4. The initial and worn profiles have been positioned according to reference planes determined outside worn areas. 
2.3 Determination of the wear coefficient

The Archard wear formulation [9] describes the wear volume as dependent on the load $F_{N}$, the sliding distance $s$, and the wear coefficient $K$. This coefficient depends on the material couple and is originally defined as the probability of contacts resulting in the removal of a particle. The wear coefficient has been measured experimentally in this study by means of a tribometer placed before the opening of the mechanical press, where the sheet coil is unrolled.

\subsubsection{Principle and description of the specific tribometer}

The tribometer developed by Makich [11] has been intended to measure a wear coefficient with specified parameters, and has been designed specially to be integrated on a production line (Fig. 5). Thus, it allows to directly test different operating conditions during the blanking tests on the mechanical press.

As illustrated in Fig. 5, the configuration of the tribometer consists in a spherical pin $(5 \mathrm{~mm}$ diameter; same material as the punch) lying on a flat sheet of the studied metal grade. A normal load $(7 \mathrm{~N})$ is applied on the pin, as the sheet translates in a continuous motion toward the mechanical press $(37 \mathrm{~mm} / \mathrm{s}$; imposed by the stroke rate).

Contrary to most of conventional tribometers such as pin-on-disc or ring-on-ring where the wear track remains the same during the tribological experiment, the open tribometer permits the rubbing surface to be constantly renewed. Therefore, the friction conditions with this tribometer get closer to the ones met during the blanking operation. Greban [12] has performed friction tests on a conventional pin-on-disc tribometer and noticed that, despite the ease of implementation of this method, the operating conditions were very different from 
those found during blanking. Contrary to the pin-on-disc tribometer, the punch always rubs into a renewed material at each stroke, allowing to better evacuate wear particles for example.

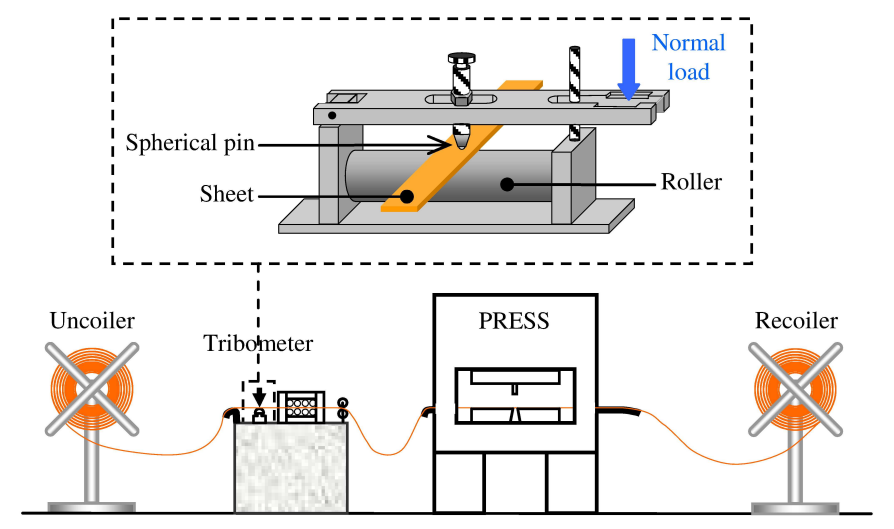

Fig. 5 : Tribometer location on the production line

After a prescribed sliding distance of metal sheet, the spherical pin has been taken apart from the tribometer and the volume loss has been measured. This procedure has been repeated two times to obtain a mean value of the volume loss.

\subsubsection{Evaluation of the wear coefficient}

After a sufficient sliding distance, the material loss of the spherical pin has appeared to be comparable to a regular spherical cap. Then, the first approach for determining the volume loss has consisted in measuring the radius of the base of the cap and using the expression of a hemisphere volume (Fig. 6a and 6b). However, a closer look at the worn pin has revealed cavities and asperities contributing significantly to the volume loss. Therefore, a second approach has been considered by employing a 3D topographic acquisition method with a confocal microscope (Alicona $\left.{ }^{\circledR}\right)$. The acquired image has been postprocessed with Gwyddion ${ }^{\circledR}$ software in order to create a reference plan, corresponding to the non-worn shape of the sphere. The wear volume has then been determined by integrating the volume under the reference plane with Surfcom® software (Fig. 7). 

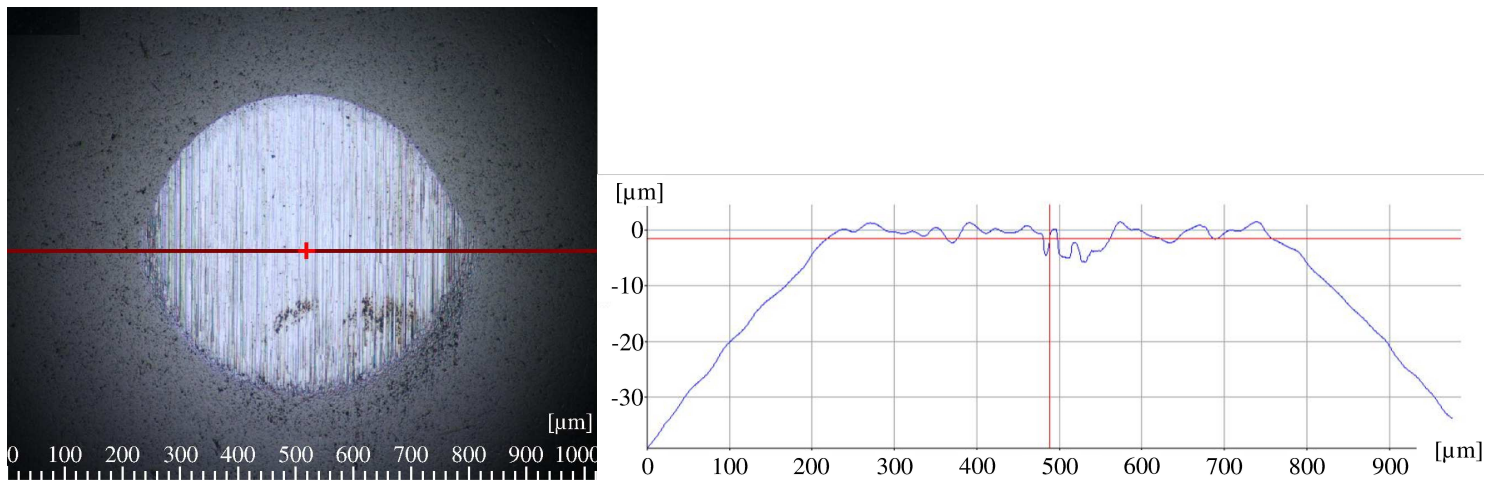

Fig. 6 : a) Wear aspect of spherical pin (2025 $\mathrm{m}$ sliding distance); b) corresponding wear profile at center line

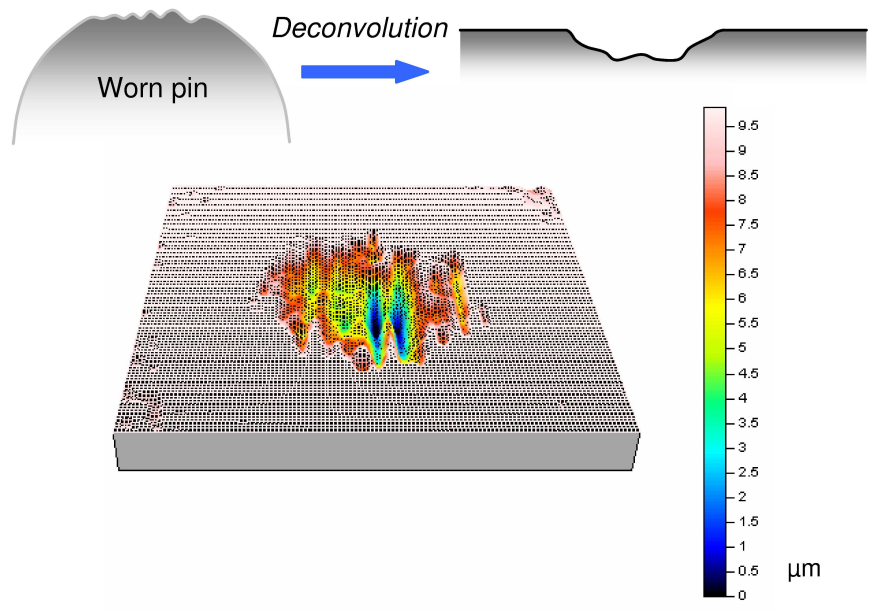

Fig. 7 : Deconvolution of the spherical shape of the pin

Finally, the wear coefficient $K$ has been calculated by means of the Archard equation [9] by dividing the volume loss of the pin $V$, by the constant applied load $F_{N}$ multiplied by the total sliding distance $s$, thus leading to a value of $2.110^{-11} \mathrm{~mm}^{2} / \mathrm{N}$ for the tungsten carbide/copper-iron alloy couple.

\section{Wear modelling}

Wear is a phenomenon widely investigated due to its economical concerns. Mechanical wear can be sorted in several classes, the most commons in metal forming being the abrasive wear and adhesive wear. In the literature, several authors [13 - 15] have reviewed numerous models to predict wear phenomena. Hegadekatte et al. [16] have classified wear models into 
two main categories: mechanistic models based on material failure mechanisms, and phenomenological models which are using principles of contact mechanics. In the first category, Suh [17] has developed the delamination theory of wear which involves dislocation mechanics. Kapoor [18] has studied wear under repeated loading where the material accumulates unidirectional plastic strain until a critical value, which leads to a failure termed ratchetting. In the second category, Archard [9] has proposed a simple linear model in terms of material volume loss, contact pressure and sliding distance of contacting parts. Over the past, Archard's equation has been modified to meet particular case studies. Rhee [19] has proposed a wear equation with separate exponents on the contact pressure and the sliding distance. Sarkar [20] has included the friction coefficient to take account of the asperity junction growth in wear phenomenon. Ersoy-Nürnberg et al. [5] have presented a wear coefficient to be variable with the accumulated wear work, which depends on dissipated energy.

The Archard model has been considered in this study to be a suitable solution in connection with a finite-element model, since each variable can be calculated within the blanking simulation. Moreover, the proposed experimental method for punch wear measurement favoured a phenomenological approach, as it was based on observing and fitting a punch shape resulting from prescribed working conditions.

\subsection{The Archard model}

The Archard wear model is widely used to predict the wear behaviour of surfaces. This model is based on a phenomenological approximation of the adhesive wear mechanism that takes into account the load and the sliding distance in the interface of two contacting bodies. The formulation has been adapted to other wear mechanisms and can be expressed as follows: 
$V=K \cdot F_{N} \cdot s$

where $V$ is the worn volume, $F_{N}$ is the applied load and $s$ is the sliding distance. The wear coefficient $K$ is originally defined as the probability that an asperity contact produces a wear particle. It is also interpreted as "the fraction of asperities yielding wear particles, as the ratio of the volume worn to the volume deformed, as a factor inversely proportional to a critical number of wear cycles, and as a factor reflecting the inefficiencies associated with the various processes involved in generating wear particles" [21]. This coefficient is established for a material couple, and can be measured experimentally by means of a tribological test (see section 2.3).

3.2 Numerical implementation of the model

By considering a representative elementary volume of material, Eq. (2) can be written:

$d V=K \cdot d F_{N} \cdot d s$

Introducing the elementary contact area $d \Omega$ allows to express the wear depth $d W$ from the relations $d V=d W \cdot d \Omega$ and $d F_{N}=p \cdot d \Omega$, where $p$ is the contact pressure:

$d W=K \cdot p \cdot d s$

For numerical applications, Eq. (4) is calculated over a time interval $\left[t_{n}, t_{n+1}\right]$ corresponding to the $n$ and $(n+1)$ increments of the simulation. The incremental expression is then implemented following an Euler scheme:

$W_{P_{i}}^{n+1}=W_{P_{i}}^{n}+K \cdot p_{P_{i}}^{n+1} \cdot\left(s_{n}^{n+1}\right)_{P_{i}} \quad$ with $\left(s_{n}^{n+1}\right)_{P_{i}}=\| s_{P_{i}}^{n+1}|-| s_{P_{i}}^{n}||$

where $W_{P_{i}}^{n}$ is the wear depth at increment $n$ calculated for a given punch node $P_{i}$ of

coordinates $\mathbf{x}_{i}$, and $p_{P_{i}}^{n+1}$ is the contact pressure at increment $(n+1)$ expressed at the same node. 
$\left(s_{n}^{n+1}\right)_{P_{i}}$ is the sliding distance between increments $n$ and $(n+1)$ and $s_{P_{i}}^{n}$ is the cumulated sliding distance at increment $n$ provided by the FE solution. This value can be negative due to the non unidirectional movement of the nodes.

However, as the punch is modelled by rigid elements, no calculation of mechanical variables is performed on this tool. Thus, the contact pressure and sliding distance values calculated on the sheet are used to determine wear on the punch. Wear depth on the punch $W_{P}$ is then obtained by integration of the calculated wear depth from the sheet $W_{T}$ along the corresponding punch profile $l$ :

$$
\left\{\begin{array}{l}
W_{P}(\mathbf{x})=\frac{1}{\phi(\mathbf{x})} \int_{l} W_{T}(\mathbf{y}) \varphi(\mathbf{x}-\mathbf{y}) \mathrm{d} \mathbf{y} \\
\phi(\mathbf{x})=\int_{l} \varphi(\mathbf{x}-\mathbf{y}) \mathrm{d} \mathbf{y}
\end{array}\right.
$$

where $\mathbf{x}$ and $\mathbf{y}$ are respectively the coordinate vector of the punch nodes and the sheet surface nodes.

According to the non-local modelling developed by Pijaudier-Cabot and Bazant [22], the product $p_{P_{i}}^{n+1} \cdot\left(s_{n}^{n+1}\right)_{P_{i}}$ defined at punch node $P_{i}$ is obtained by calculating the weighted average values of the product $p_{T_{j}}^{n+1} \cdot\left(s_{n}^{n+1}\right)_{T_{j}}$ obtained from the sheet nodes $T_{j}$ (coordinates $\mathbf{y}_{j}$ ) and located near the considered punch node. Eq. (5) can then be expressed by using Eq. (6) as a discrete integral corresponding to the calculation of the wear depth at node $P_{i}$ :

$$
\begin{aligned}
& W_{P_{i}}^{n+1}=W_{P_{i}}^{n}+\frac{N_{P} \cdot K}{\phi\left(\mathbf{x}_{i}\right)}\left[\sum_{j=1}^{N} p_{T_{j}}^{n+1} \cdot\left(s_{n}^{n+1}\right)_{T_{j}} \cdot \varphi\left(\mathbf{x}_{i}-\mathbf{y}_{j}\right)\right] \\
& \text { with } \phi\left(\mathbf{x}_{i}\right)=\sum_{j=1}^{N} \varphi\left(\mathbf{x}_{i}-\mathbf{y}_{j}\right) \text { and } \varphi\left(\mathbf{x}_{i}-\mathbf{y}_{j}\right)=\exp \left[-\left|\mathbf{x}_{i}-\mathbf{y}_{j}\right|^{2} / L_{c}^{2}\right]
\end{aligned}
$$


where $L_{c}$ is the parameter which determines the number of sheet nodes $N$ considered for the weighting operation ( $L_{c}=2 t_{P}$ in this study) and $N_{P}$ is the number of punch strokes. This distribution allows to take account of the relative distance separating a given punch node from a sheet node, thus traducing a more realistic wear behaviour.

The wear prediction is performed during the steady-state wear stage. Therefore, a multiplication of the wear depth from one punch stroke is appropriate to approximate the wear occurring after several punch strokes. However, this number of strokes should not be too high for two main reasons. The first one is that one must avoid reaching the accelerated wear state. The second reason is that according to the shape variation of the wear profile during the process, stress and contact pressure fields are expected to vary, thus modifying wear distribution along the punch profile. Choosing an elevated number of punch strokes will not take into account this wear distribution variation and will lead to a significantly different wear profile.

The wear algorithm based on these equations has been programmed under Matlab ${ }^{\circledR}$ environment. Data including contact pressure, relative tangential motion, coordinates of the sheet and punch nodes, have been collected from the FE simulation. After processing, punch worn profiles have been built by normal projection of wear depth values.

\section{Numerical simulation}

4.1 Modelling of the blanking process

\subsubsection{Model main characteristics}

The process modelling has been performed with the FE code Abaqus/CAE 6.9®, using the large deformation theory. The solution has been obtained by the included implicit solver Abaqus/Standard ${ }^{\circledR}[23]$, following a quasi-static approach of the process simulation. The Newton iteration method has been employed to solve the nonlinear equilibrium equations. 
Due to the revolution geometry of the problem, axisymmetry has been considered for the whole setup, so only one meridian plane of the problem has been modelled.

The blanking tools have been modelled using 1D rigid elements. The sheet has been mainly meshed with quadrangular first order axisymmetric elements. The real worn punch geometry has been fitted by an exponential formula in order to improve the accuracy of the numerical modelling (Fig. 4).

In the blanking process, deformations are concentrated in the shear band located between the punch and die cutting edges. Thus, the sheet mesh has been divided in three zones to obtain a fine mesh in the zone of large deformation, and a coarse mesh elsewhere to minimize simulation computational cost. Moreover, fine mesh has been required on the sheet surface to obtain accurate contact pressure and sliding distance data necessary for wear calculation (Fig. 8b).

The sheet material behaviour has been described by an elasto-plastic Von Mises constitutive model with isotropic hardening, and the stress-strain data necessary for the non-linear behaviour of the sheet has been obtained by Makich [11] using tensile tests.
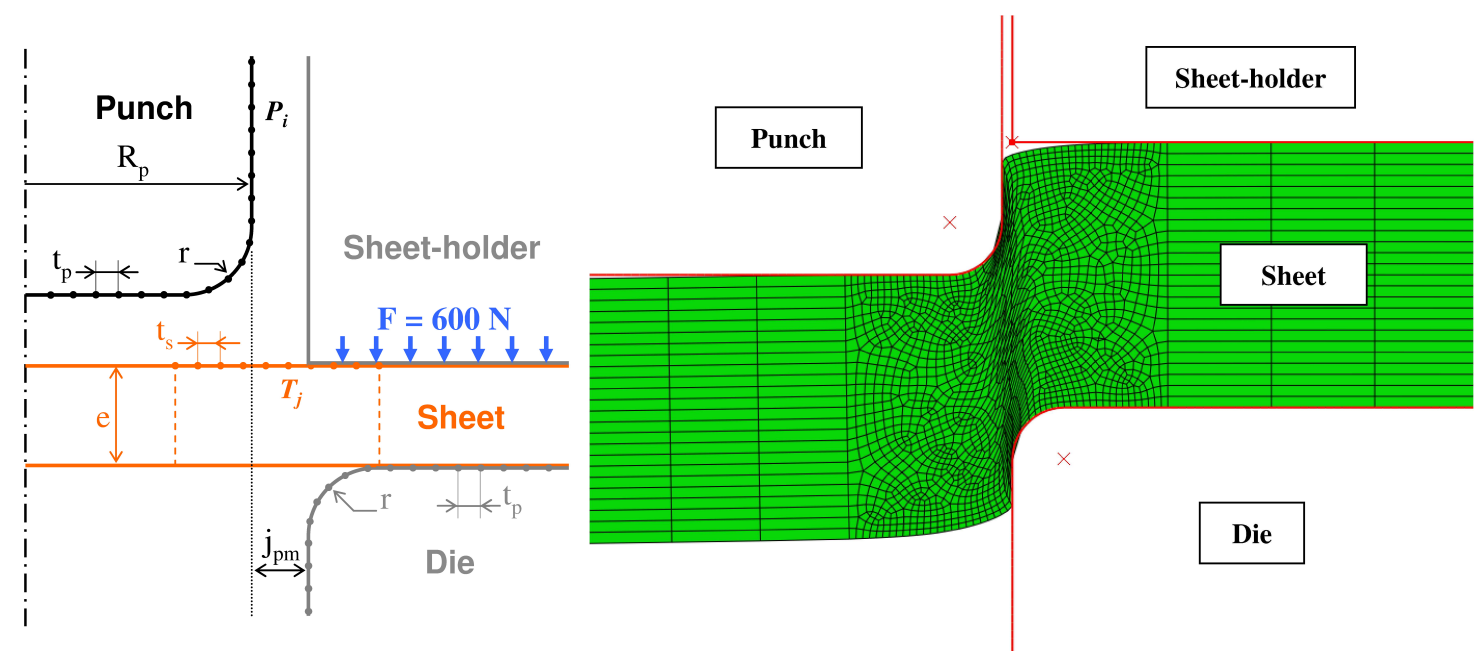

Fig. 8. a) Axisymmetric blanking scheme b) Deformed mesh at $50 \%$ of punch penetration 


\subsubsection{Contact formulation}

Contact between the die, the sheet and the sheet-holder has been modelled by Coulomb law with a value of 0.1 for the friction coefficient. Due to the lubrication applied during the blanking process, no friction has been considered between the punch and the sheet.

For the contact formulation, the penalty contact algorithm has been used by defining a master surface (punch profile) and a slave surface (on sheet boundary).

\subsubsection{Failure}

Examination of the experimental blanked edge generally reveals two main zones traducing the material behaviour during the process. The first one is the sheared zone, formed by the punch before failure occurs. The second one is the fracture zone and corresponds to the ductile fracture of the material as the punch continues to penetrate the sheet. Failure was not taken into account in the numerical model because it is supposed that only wear on the punch lateral face is involved after the onset of material failure. This hypothesis is suggested by the observations made by Maeda [2] and Faura [24] concerning the face wear of punch, which is less developed than flank wear. Indeed, blanking vertical force drops off when failure is initiated, reducing the contact pressure on the punch face; contact between the punch face and the sheet is also lost when the part or scrap is separated from the sheet. However, failure mechanism will decrease the stresses within the material and will create new contact surface, thus influencing wear results especially on punch flank.

The maximum punch penetration (relative percentage of the sheet thickness) has been defined according to the experimental data of the shear zone height and fracture zone height of the sheet, which remained rather constant during the steady-state wear stage.

As mentioned in Eq. (5), the computation of pressure and sliding distance has been required to calculate wear. Contact pressure in Abaqus ${ }^{\circledR}$ is defined as the normal stress between two faces at a point, and depends on the interpenetration of the surfaces. The sliding distance is 
provided by the relative tangential motion, which is defined as the scalar product of the slip direction and the incremental relative nodal displacement vector. This vector measures the motion of a sheet node relative to the motion of the punch. The sliding distance computed is thus direction-dependent and cumulated at each increment.

\subsubsection{Remeshing}

In metal forming processes, one of the major problems to deal with is the non linearities due to the large displacements and deformations. Some finite elements of the mesh can become severely distorted and no longer provide a good discretization of the problem. The remeshing solution retained in this work has consisted in creating a new mesh before the distortion of some elements became too important, and to transfer the mechanical variables field from the old deformed mesh to the new one. After the remeshing operation, a loss of structure equilibrium has been obtained so that several increments have been necessary to reach a new equilibrium state.

\section{Results and discussion}

Firstly, the punch has been modelled according to the experimental punch dimensions except for the punch cutting edge which has been approximated by a constant quarter circle. Secondly, experimental wear profiles from the method presented in section 2.2 .3 have been employed to improve the cutting edge wear modelling and trying to predict worn profiles. Thirdly, a global discussion is presented at the end of this section. 


\subsection{Setup test of the wear algorithm}

Since the value of the punch edge radius is closely related to the tool wear status [25], a first approach for testing the wear algorithm has consisted in varying the edge radius in the FE simulation and observing the effects on the punch wear distribution. In a first attempt, the size chosen for the radii has been sufficiently large to allow a significant penetration of the punch without remeshing the sheet. Indeed, a small radius requires small enough elements to avoid interpenetration of the sheet into the punch surface, but small elements will also degenerate more rapidly compared to larger elements for a given punch displacement. According to the dimension of each punch edge radius, the punch elements size has been adapted to always keep 10 elements within the quarter circle for accuracy purpose.

Thus, the setup test has been performed with respect to the geometrical dimensions listed in Table 1 and by varying the radius of curvature at the punch cutting edge from a range of values $(25 \mu \mathrm{m}, 50 \mu \mathrm{m}$ and $65 \mu \mathrm{m})$. Die wear has not been taken into account during the experimental study, so the hypothesis has been made that it worn the same manner as the punch.

The wear coefficient for this study has been fixed to $2.10^{-3} \mathrm{~mm}^{2} / \mathrm{N}$ in order to get a significant quantitative wear depth for one punch stroke.

The results of wear depth provided by the algorithm have been originally expressed along the curvilinear abscissa of the punch profile (Fig. 9). This representation gives a global view of wear but is not appropriate for comparing profiles. Thus, a nondimensionalized curvilinear abscissa based on the extremities of the edge radius has been introduced. As shown in Fig. 10, the middle of the quarter circle corresponds to the zero abscissa (point $O$ ), and the left and right extremities respectively represent the "-1" (point $A$ ) and "+1" (point $B$ ) abscissa. The nondimensionalized curvilinear abscissa of point $M$ is then calculated by dividing the arc 
length $O M$ by $O A$ (which corresponds to an eighth of the circle defined by the radius of curvature $r$ ).

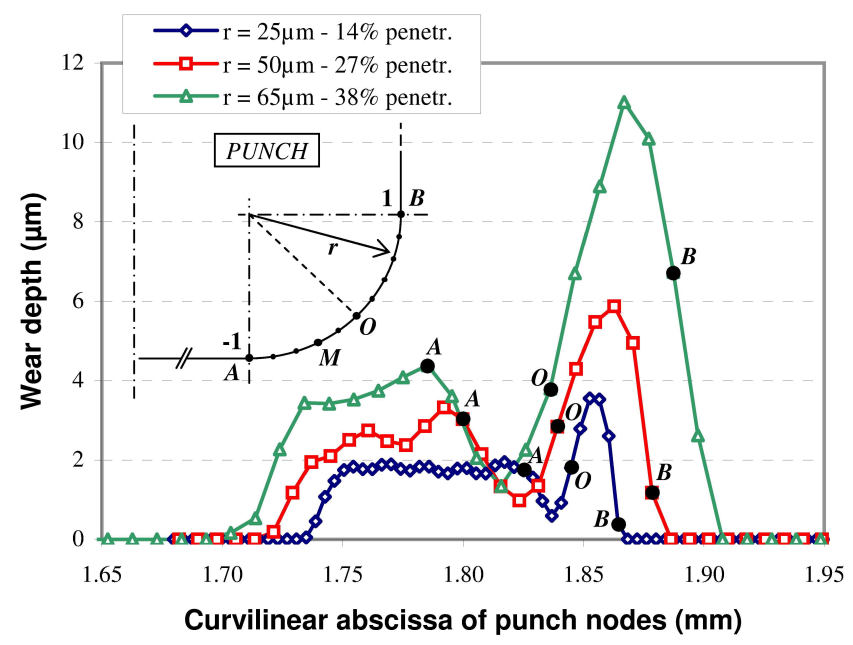

Fig. 9. Wear distribution for three punch edge radii at different punch penetrations

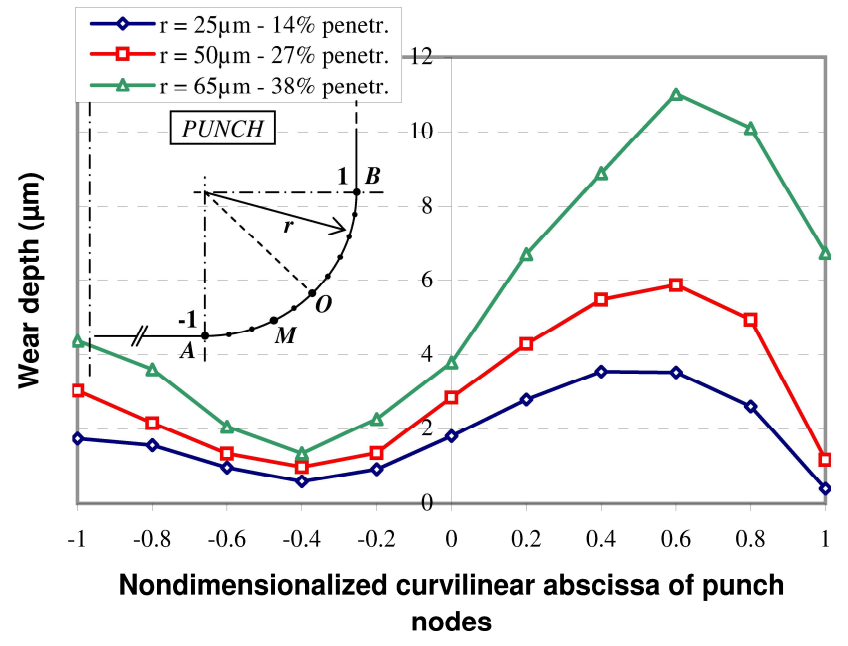

Fig. 10. Nondimensionalized curvilinear abscissa scheme and wear distribution in the edge radius

This first approach has revealed the three redundant types of wear observed along the punch profile for every performed simulation. As shown in Figs. 9 and 10, the punch face tends to wear slowly and rather steadily. Then, wear depth drops off in the lower part of the cutting edge radius to suddenly increase on the punch flank. Wear depth continues to grow as the punch penetrates the sheet. The location of the depicted zones remains the same during the penetration of the punch. These three types of wear are illustrated by the wear kinetics, corresponding to the evolution of the wear depth during the punch penetration (Fig. 11.). 


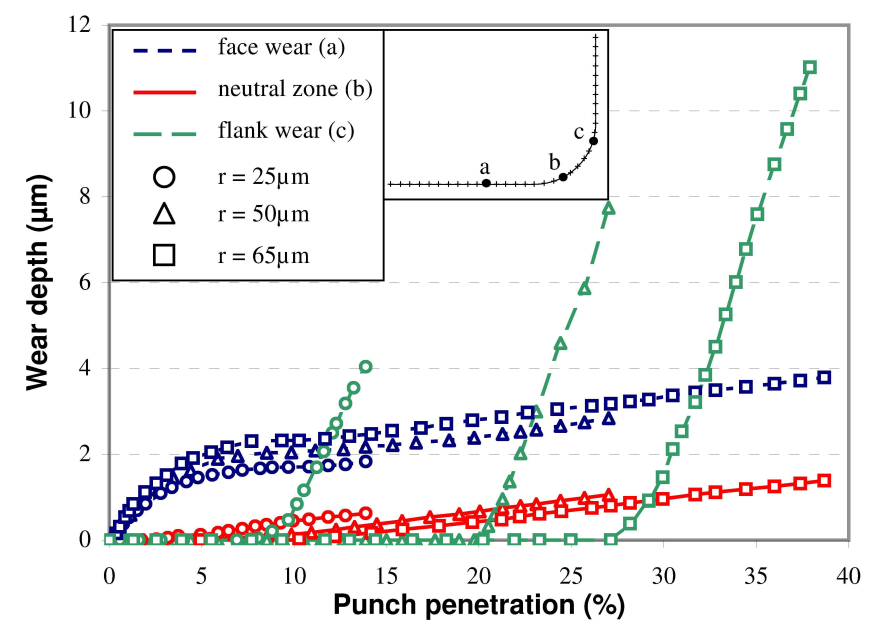

Fig. 11. Wear kinetics at three representative locations of the punch

The region of minimal wear (point $b$ in Fig. 11 and abscissa value of -0.4 in Fig. 10) has been identified as a "neutral" zone where the punch and the sheet surfaces move similarly, thus conducting to a small relative displacement. Contact pressure at this location tends to reach a local maximal value but this value keeps lower than the pressures under the punch.

The resulting wear is then less important at this place than elsewhere. Moreover, this "neutral zone" is located at the same nondimensionalized abscissa for every punch cutting edge radius.

\subsection{Wear prediction from real punches}

Once the algorithm has been tested on setup test, two worn punch profiles obtained during the experimental study have been integrated into the FE modelling. The aim has been to predict the shape of the worn geometry by calculating wear depth and multiplying the results by a determined punch strokes number. Thus, the mesh has been applied on the punch geometry defined by the fitting curve presented on Fig. 4. The first one corresponds to 766,500 punch strokes and the second one 1,266,500. For numerical concerns, these profiles have been chosen sufficiently worn (i.e. with a large curvature radius) and still in a steady-state wear to 
allow a significant penetration of the punch, and then reduce the number of remeshing to be performed. Fig. 12 depicts the blanking force - punch penetration curves obtained with the studied profiles.

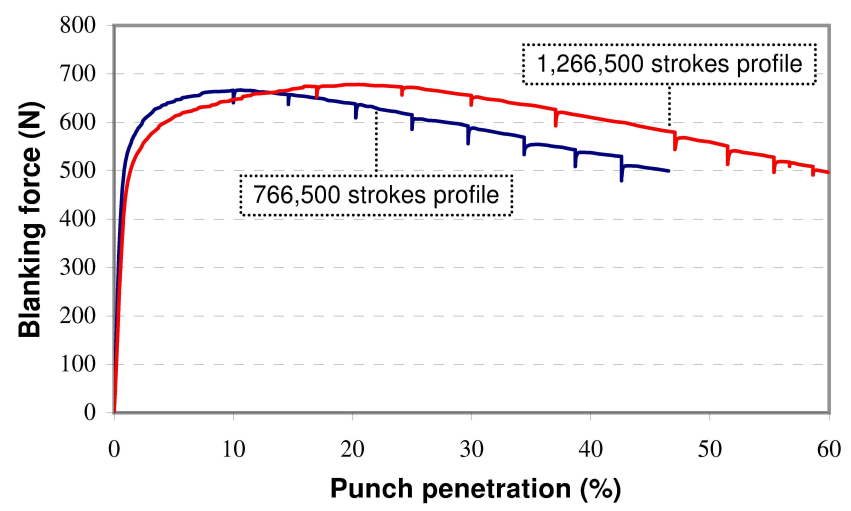

Fig. 12. Blanking force versus punch penetration for two punch profiles

Discontinuities on each curve correspond to the different remeshing steps performed during the simulation. It can be seen that the maximum force appears for a more important penetration and is higher for the most worn profile. This results correlates with those obtained by Choy and Balendra [26].

Wear calculation has then been performed starting from a worn geometry and by taking account of the experimental wear coefficient $\left(K=2 \cdot 1 \cdot 10^{-11} \mathrm{~mm}^{2} / \mathrm{N}\right)$. Fig. 13a represents the evolution of wear depth at each punch node from the first worn profile (766,500 strokes), during one punch penetration. 
a)

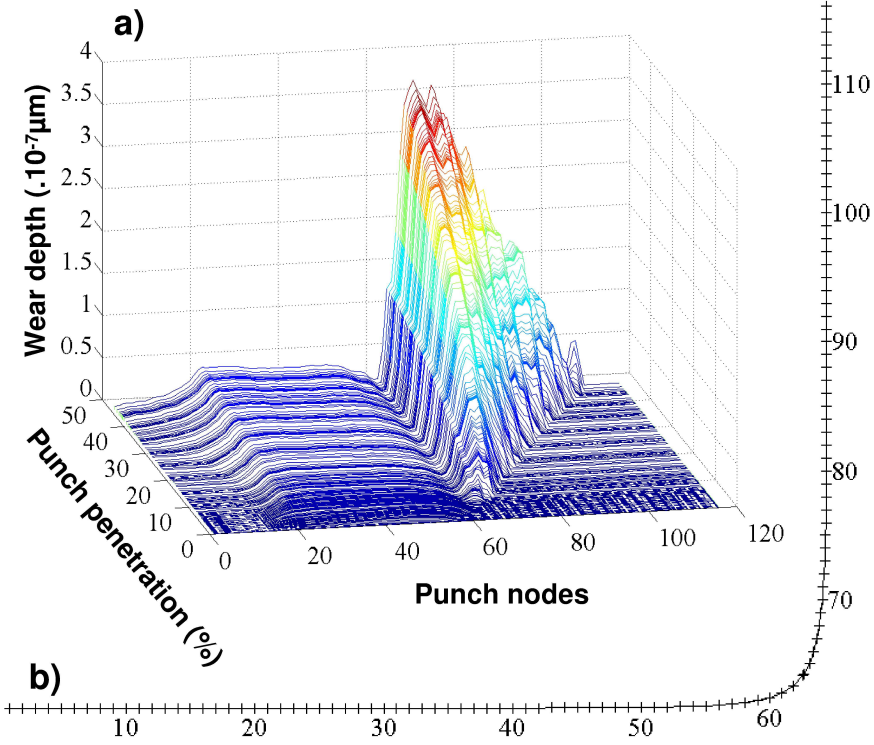

Fig. 13. (a) Wear evolution from worn profile at 766,500 strokes (b) Corresponding punch profile with node numbering

Again, it can be noticed that the location of the three different types of wear remains the same during the penetration. Concerning punch flank, wear propagates as new contacts between the punch and the sheet are established. For example, wear depth at node 80 starts from a punch penetration of 20\%. As shown in Fig. 14 for two punch nodes (nodes 30 and 67), wear evolution at different locations of the punch has been sufficiently consistent to extrapolate the wear depths to a punch penetration of $70 \%$ (according to the experimental shear zone height). The extrapolation has been performed using a logarithmic law of the form $y=a \cdot \ln (x)+b$ with $a>0$ and $b<0$.

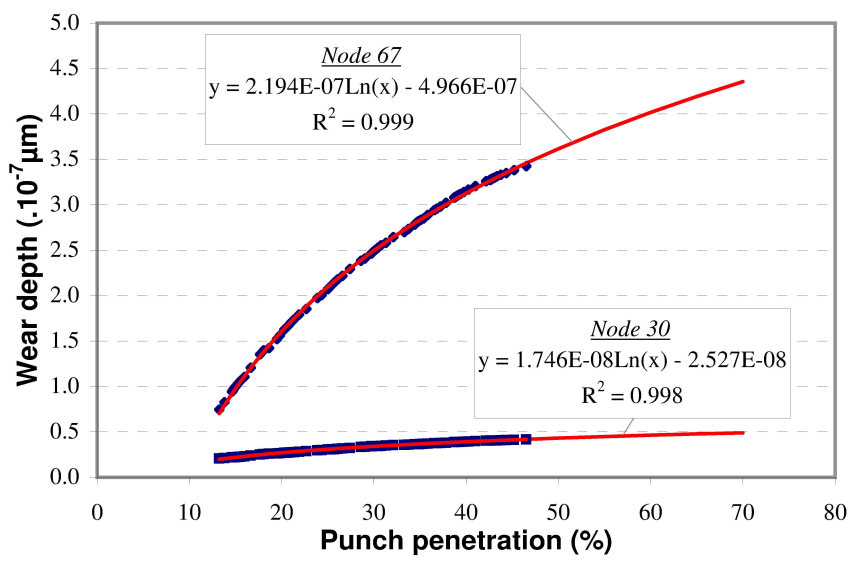

Fig. 14. Wear evolution for punch nodes $n^{\circ} 30$ and $n^{\circ} 67$ from 766,500 strokes profile 
Extrapolated values of wear depth at each punch node have then been reported on the corresponding normal vectors to generate worn profiles. The value $N_{P}$ from Eq. (7) has been set to 37,500 punch strokes in order to compare the numerical results with the experimental corresponding profile (see Fig. 15).

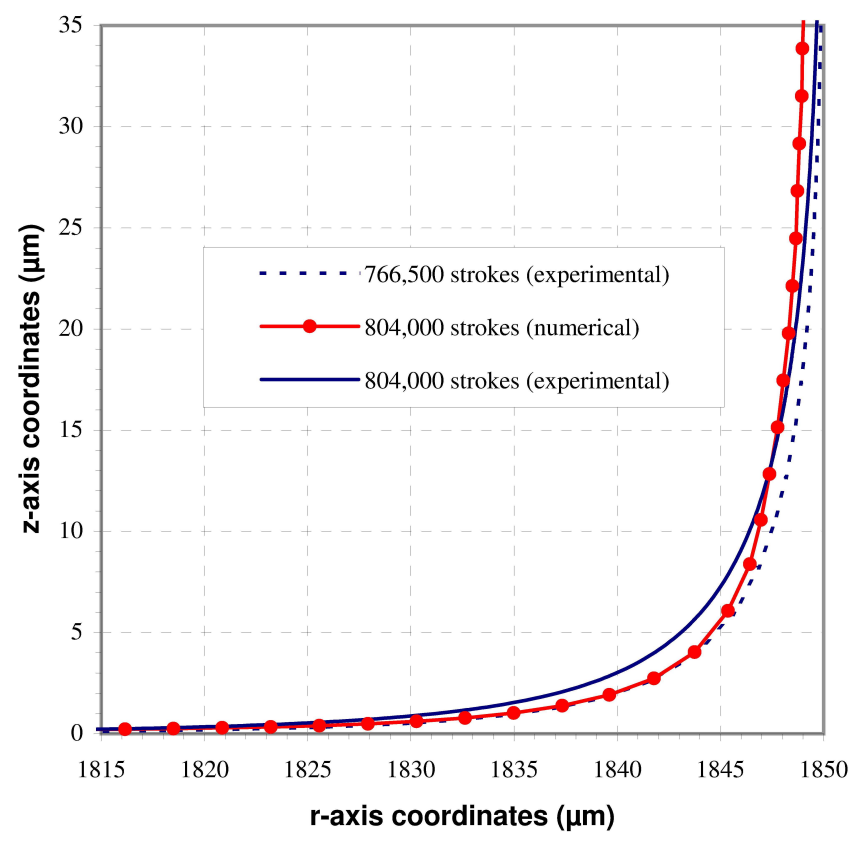

Fig. 15. Worn profiles at 766,500 and 804,000 punch strokes

For the numerically predicted worn profile at 804,000 punch strokes (Fig. 15), a magnification factor of 50 has been applied to the computed wear values. This has been performed to clearly display the computed profile despite the low values of wear depth.

\subsection{Discussion}

Numerically predicted worn profiles show most wear on the punch flank. Wear depth values in the cutting edge radius are less significant due to the presence of a neutral zone. However, wear is globally less important than the one observed experimentally. 
Different causes of the non-correspondence between experimental and theoretical results may be identified.

- The Archard phenomenological wear formulation neglects the tribological aspect of wear (surface roughness, third-body, ...) by focusing mainly on the sliding distance and contact pressure effect. It can be appropriate to determine wear for simple interactions as in a tribometer system (constant load and sliding speed) but shows some limitations when applied in situations with varying parameters.

- The wear coefficient determined by the specific tribometer shows lower values compared to bibliography. According to [27], the wear coefficient $K$ varies over the range $10^{-2}-10^{-}$ ${ }^{6} \mathrm{~mm}^{2} / \mathrm{N}$ under unlubricated conditions for different material couples. This difference can be partly explained by the configuration of the tribometer which differs from conventional ones where wear particles are not evacuated and contribute to the wear mechanism.

- The fitted punch profile based on experimental data allows to obtain a smooth FE mesh of the punch, but in counterpart it neglects the surface roughness of the tool, which can take an important role in the wear mechanism. Indeed, the surface asperities create local stress concentrations which deform the metal plastically and lead to the formation of wear particles.

- The fitting curve used to model the experimental wear profile is more accurate than a constant curvature radius. However, the formulation can locally misrepresent the punch cutting edge.

- One simulation of blanking is performed and then the wear results are multiplied by the number of punch strokes. A too important value may have favoured the neutral face wear and misrepresent the real evolution of wear.

- Only the down travel of the punch has been modelled. It has been observed experimentally that after the separating of the cut part, the sheet tends to pinch the punch 
due to remaining elastic stress. This contributes significantly to the flank wear during the up travel of the punch.

\section{Conclusion}

The experimental study carried out by Makich [11] has provided sufficient data to establish and adjust a punch wear algorithm for blanking of copper alloy thin sheet. First, a specific tribometer has allowed to test the material couple in a configuration closer to the conditions met during the blanking process. Second, an original double-print method for wear profiles extraction has shown interest in accurately modelling the punch geometry. The Archard formulation used to model the abrasive wear mechanism in blanking process has given a qualitative description of punch wear compared to the experimental observations. Third, an original method has been developed to transfer the wear calculation from the sheet nodes to the punch geometry. Finally, the combination of experiments with FE simulations of the process allows to explain the wear distribution along the punch profile, and to exhibit a minimal wear zone in the cutting edge radius. To improve the wear modelling especially on the punch flank, ductile fracture and crack propagation should be covered by further research.

\section{Acknowledgements}

The authors wish to thank the industrial partners Alstom, Diehl Augé Découpage, Cryla, CETIM, and financial partners Région Franche-Comté, FEDER, Conseil Général du Doubs 
and Communauté d'Agglomération du Grand Besançon, which have made this research work possible.

\section{References}

[1] T. Maeda, K. Matsuno, Wear on shearing tools, J. Soc. Mech. Eng. 10 (1967) 197-205.

[2] T. Maeda, I. Aoki, The study on wear of blanking tool for 18-8 stainless steel and bainite hardened steel strips, J. Faculty Eng., Univ. Tokyo (B) XXXII (1974) 443-475.

[3] J.J. Hernandez, P. Franco, M. Estrems, F. Faura, Modelling and experimental analysis of the effects of tool wear on form errors in stainless steel blanking, J. Mater. Process. Tech. 180 (2006) 143-150.

[4] G. Monteil, F. Gréban, X. Roizard, In situ punch wear measurement in a blanking tool by means of thin layer activation, Wear 265 (2008) 626-633.

[5] K. Ersoy-Nürnberg, G. Nürnberg, M. Golle, H. Hoffmann, Simulation of wear on sheet metal forming tools - An energy approach, Wear 265 (2008) 1801-1807.

[6] M.R. Jensen, F.F. Damborg, K.B. Nielsen, J. Danckert, Applying the finite-element method for determination of tool wear in conventional deep-drawing, J. Mater. Process. Technol. 83 (1998) 98-105.

[7] B.-A. Behrens, F. Schaefer, Prediction of wear in hot forging tools by mean of finiteelement-analysis, J. Mater. Process. Tech. 167 (2005) 309-315.

[8] R. Hambli, Blanking tool wear modelling using finite element method, Int. J. Mach. Tools Manuf. 41 (2001) 1815-1829.

[9] J.F. Archard, Contact and rubbing of flat surfaces, J. Appl. Phys. 24 (1953) 981-988. 
[10] R. Hambli, BLANKSOFT : a code for sheet metal blanking processes optimization, J.

Mater. Process. Tech. 141 (2003) 234-242.

[11] H. Makich, Etude théorique et expérimentale de l'usure des outils de découpe; Influence sur la qualité des pièces découpées, Ph.D. Thesis, University of Franche-Comté, Besançon, France (2011).

[12] F. Gréban, Découpabilité du cuivre et des alliages cuivreux, Ph.D. Thesis, University of Franche-Comté, Besançon, France (2006).

[13] H. C. Meng, K. C. Ludema, Wear models and predictive equations: their form and content, Wear 181-183 (1995) $443-457$.

[14] P. J. Blau, Fifty years of research on the wear of metals, Tribol. Int. 30 (1997) $321-331$.

[15] S. M. Hsu, M. C. Shen, A. W. Ruff, Wear prediction for metals, Tribol. Int. 30 (1997) $377-383$.

[16] V. Hegadekatte, S. Kurzenhäuser, N. Huber, O. Kraft, A predictive modeling scheme for wear in tribometers, Tribol. Int. 41 (2008) $1020-1031$.

[17] N.P. Suh, The delamination theory of wear, Wear 25 (1973) $111-124$.

[18] A. Kapoor, Wear by plastic ratchetting, Wear 212 (1997) 119 - 130.

[19] S. K. Rhee, Wear equation for polymers sliding against metal surfaces, Wear 16 (1970) $431-445$.

[20] A. D. Sarkar, Friction and wear, Academic Press, London (1980).

[21] D.A. Rigney, The roles of hardness in the sliding behaviour of materials, Wear 175 (1994) 63-69. 
[22] G. Pijaudier-Cabot, Z.P. Bazant, Nonlocal damage theory, J. Eng. Mech.-ASCE 113 (1987) 1512-1533.

[23] ABAQUS/Standard-HKS, Abaqus Analysis User's Manual - Version 6.9.

[24] F. Faura, J. Lopez, J. Sanes, Criterion for tool wear limitation on blanking 18-8 stainless steel strips, Rev. Metal. Madrid 33 (5) (1997) 304-310.

[25] R. Hambli, Etude expérimentale, numérique et théorique du découpage des tôles en vue de l'optimisation du procédé, Ph.D. Thesis, ENSAM, Angers, France (1996) 80-92.

[26] C.M. Choy, R. Balendra, Experimental analysis of parameters influencing sheared-edge profiles, In Proc. of the $4^{\text {th }}$ Int. Conf. on Sheet Metal (1996) 101-110.

[27] S. Kalpakjian, Manufacturing processes for engineering materials, fourth ed., Prentice Hall, New Jersey, 2002. 